Case Report

\title{
Successful Treatment of Recurrent Pulmonary Mucormycosis in a Renal Transplant Patient: A Case Report and Literature Review
}

\author{
Morgan S. Martin, ${ }^{1}$ Alison A. Smith, ${ }^{1}$ Monica Lobo, ${ }^{2}$ and Anil S. Paramesh ${ }^{3}$ \\ ${ }^{1}$ Department of Surgery, Tulane University School of Medicine, New Orleans, LA, USA \\ ${ }^{2}$ Department of Pathology, Tulane University, School of Medicine, New Orleans, LA, USA \\ ${ }^{3}$ Tulane University School of Medicine, Tulane Abdominal Transplant Institute, New Orleans, LA, USA \\ Correspondence should be addressed to Alison A. Smith; alison.annette.smith@gmail.com
}

Received 16 November 2016; Revised 21 February 2017; Accepted 2 March 2017; Published 13 March 2017

Academic Editor: David Conti

Copyright (C) 2017 Morgan S. Martin et al. This is an open access article distributed under the Creative Commons Attribution License, which permits unrestricted use, distribution, and reproduction in any medium, provided the original work is properly cited.

\begin{abstract}
Background. We describe the unusual case of a recently transplanted cadaveric renal transplant recipient who presented with recurrent pulmonary mucormycosis. Case Report. An 18-year-old man with end stage renal disease secondary to congenital renal agenesis status after cadaveric kidney transplant 4 months before presented with acute onset of fever, hemoptysis, and back pain. The patient underwent an emergent left lower lobectomy due to the critical nature of his illness. He was also treated with amphotericin with resolution of his symptoms. One week later, he had evidence of recurrent disease on imaging with a surgical site infection. He underwent reexploration with evacuation of an empyema and debridement of a surgical site infection. He was continued on IV antifungal therapy with isavuconazonium and amphotericin. Radiographic clearance of disease with three months of treatment was apparent with no evidence of recurrence at seven-month follow-up. Discussion. Opportunistic infections in solid organ transplant patients represent a significant source of morbidity and mortality. Most patients are treated with prophylactic anti-infective agents. However, rare infections such as pulmonary mucormycosis remain a risk. The transplant physician must be aware of these uncommon infections and their treatment strategies, including the management of uncommon recurrent disease.
\end{abstract}

\section{Introduction}

Mucormycosis, which includes fungi in the Mucorales order such as Rhizopus and Mucor, represents a rare but aggressive infection of immunosuppressed patients. Less than one percent of solid organ transplant patients are infected; however mortality can exceed greater than $80 \%$ [1-5]. Rhinocerebral, pulmonary, gastrointestinal, and disseminated disease are the most common sites for infection [6].

Most infections occur within the first year of solid organ transplant [7]. The vast majority of patients infected by mucormycosis are male, older than age of 40 with multiple risk factors, including neutropenia, diabetes, active malignancy, and iron overloaded states. In particular, renal transplant recipients are at even high risk given the presence of comorbid diabetes, use of immunosuppressants, the frequent use of voriconazole and echinocandins to treat fungal infections in transplant patients, and uremia [3, 8]. Pulmonary mucormycosis is the most common site of infection for renal transplant recipients [9]. In addition, the southern United States has the highest incidence of mucormycosis compared to other regions of the country [5].

Mucorales have known resistance to caspofungin and voriconazole. Accordingly, amphotericin B is regarded as the first-line therapeutic agent for mucormycosis [10]. However, the use of amphotericin B usually results in renal injury and concomitant loss of the renal allograft. Use of posaconazole has been proposed in several studies; however it has not shown to be as effective as amphotericin B [11]. Thus, emerging treatment strategies have included extended spectrum azoles with specific activity against Mucorales with improved side effect profiles $[12,13]$.

Surgical debridement is essential for effective treatment of mucormycosis. Due to the aggressive behavior of this fungus in immunocompromised patients, extensive surgical debridement has appeared to be necessary in successful 


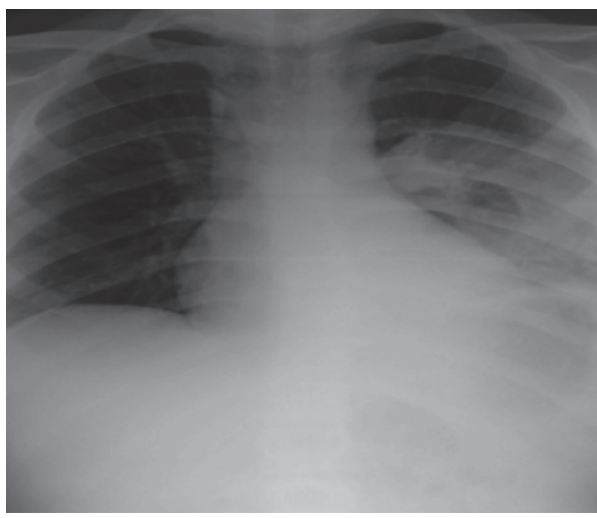

(a)

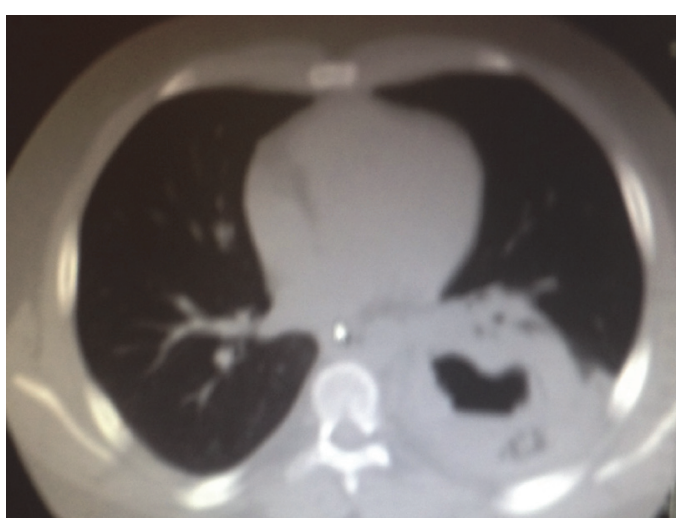

(b)

FIGURE 1: (a) Initial chest X-ray on initial presentation for an 18-year-old man status after cadaveric kidney transplant demonstrating increased opacification of the mid and lower left lung fields. (b) Computerized topography of chest/thorax for the same patient demonstrating a left lobe cavitary lesion.

clearance of disease reported in various case series $[3,14$, 15]. A review of the literature did not identify any studies on the recommended treatment of recurrent pulmonary mucormycosis.

We report the unusual case of a renal transplant recipient with refractory pulmonary mucormycosis. The objective of our study is to describe the management of recurrent lung mucormycosis in a recent cadaveric renal transplant recipient. This case report adds to the growing body of literature surrounding this phenomenon and to elucidating effective treatment algorithms.

\section{Case Presentation}

An 18-year-old man with history of end stage renal disease secondary to congenital renal agenesis presented to the Emergency Department with an acute onset of hemoptysis, back pain, and fever. He had undergone a cadaveric kidney transplant four months before at a different institution. The transplant was performed without any complications, and the patient had reportedly normal kidney function. He was taking cyclosporine, mycophenolate, and prednisone for maintenance immunosuppression. He was not on any antifungal prophylaxis. The patient was not residing in a house with routine expose to soil and livestock. He denied any weight loss or recent foreign travel. His laboratory values were significant for leukocytosis with a white blood cell count of $21,900 / \mathrm{mm}^{3}$ and elevated creatinine. His initial chest X-ray demonstrated a consolidation of the left lower lobe (Figure 1(a)). Further imaging on chest CT showed a left lower lobe cavitary lesion (Figure 1(b)). Sputum, bronchoalveolar lavage, and blood cultures were all negative for infectious etiology. His workup was additionally negative for any additional infectious etiology. His renal biopsy showed no evidence of rejection or active infection. He was initially started on broad-spectrum antibiotics and antifungals. His immunosuppressants were held.
He was taken for an open thoracotomy with a left lower lobectomy one week after initial presentation due to the critical nature of his illness. Intraoperatively, he was found to have a solitary consolidated fungal mass surrounded by an area of hemorrhagic necrosis (Figure 2). Final pathology showed Rhizopus species (Figure 3). Treatment was initiated with IV liposomal amphotericin B $50 \mathrm{mg}$ daily.

The patient demonstrated overall clinical improvement. However, one week postoperatively, the patient developed a new consolidation of his left upper lobe on chest X-ray. Repeat chest CT demonstrated an empyema. The patient also had Rhizopus cultured from his surgical site. He remained febrile with significant leukocytosis. He was taken to the operating room for an evacuation of an empyema and debridement of soft tissue surrounding the incision (Figures 4(a) and 4(b)).

Despite aggressive surgical management of his recurrent disease, the patient continued to remain symptomatic. However, he had evidence of consolidations in both lungs (Figure 5). It was then decided to add isavuconazonium (372 mg daily). The patient improved clinically, and he was discharged home 24 days after his initial presentation.

The patient was maintained on the regimen of amphotericin B (50 mg daily) and isavuconazonium (372 mg daily) after discharge. His follow-up chest X-ray 7 months after treatment showed no evidence of residual disease. His medications were discontinued at this time. To our knowledge, he has had no further issues.

\section{Discussion}

Mucormycosis is a rare infection with a high likelihood of devastating outcomes in immunocompromised patients. Currently, there is a paucity of data on optimal treatment strategies. In particular, there are no clinical studies that demonstrate successful treatment of patients with recurrent mucormycosis. Our case report demonstrates a strategy to combat recurrent mucormycosis using salvage therapy 


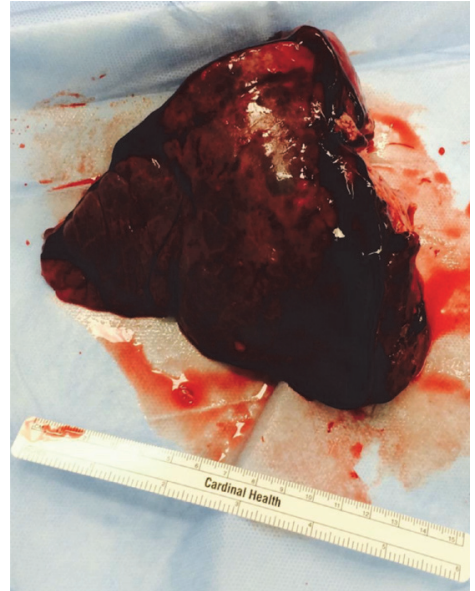

(a)

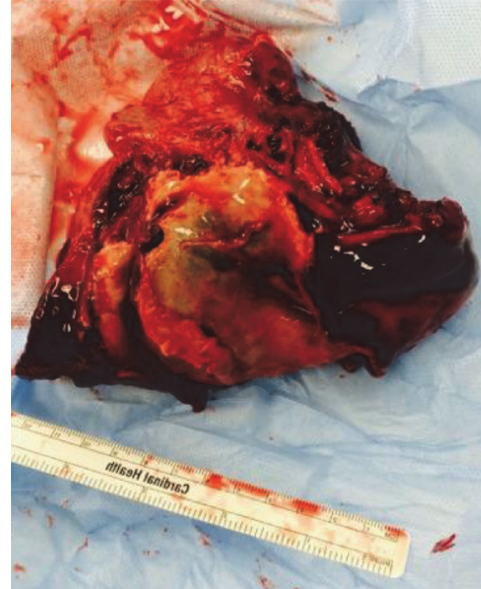

(b)

FIGURE 2: Gross pathology of consolidated fungal mass removed from lung of an 18-year-old male status after cadaveric kidney transplant. (a) Anterior and (b) posterior views of left lower lobe demonstrating a large consolidated fungal mass surrounded by area of hemorrhagic necrosis.

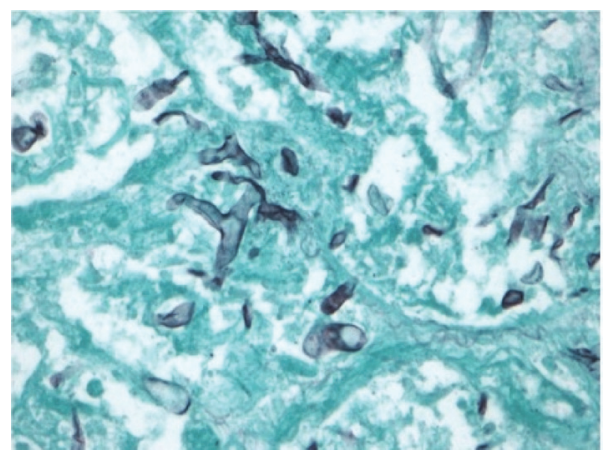

FIGURE 3: Grocott-Gomori methenamine-silver (GMS) stained tissue section of resected lung from an 18-year-old with recent kidney transplant highlighting aseptate hyphae morphologically resembling fungal class Zygomycetes (400x).

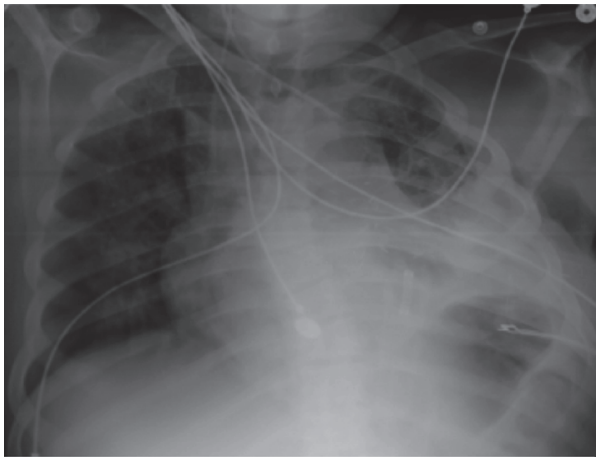

(a)

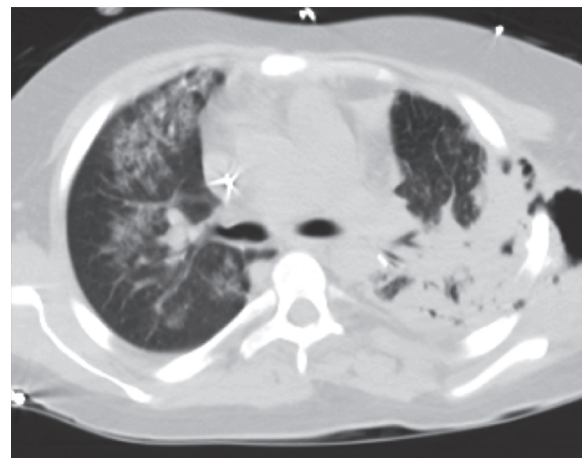

(b)

FIGURE 4: 18-year-old male renal transplant recipient 24 days status after resection of left lower lobe for a fungating mass with (a) chest X-ray demonstrating left upper lobe consolidation and (b) chest computerized topography scan showing consolidation of right upper, middle, and lower lobes. 
TABLE 1: Summary of existing publications on mucormycosis in renal transplant recipients.

\begin{tabular}{|c|c|c|c|c|}
\hline Reference & Number of patients & $\begin{array}{c}\text { Organ } \\
(N)\end{array}$ & Treatment & $\begin{array}{c}\text { Mortality } \\
N(\%)\end{array}$ \\
\hline Fisher et al., 1980 & 1 & Skin (1) & AmpB, surgery & $1 / 1(100)$ \\
\hline Nampoory et al., 1996 & 2 & Lungs (1); allograft (1) & AmpB, fluconazole & - \\
\hline Bakshi and Volk, 2001 & 1 & Lungs (1) & AmpB & $0 / 1(0)$ \\
\hline Godara et al., 2011 & 16 & $\begin{array}{c}\text { Rhino (9); lungs (5); } \\
\text { allograft (1); disseminated } \\
\text { (1) }\end{array}$ & AmpB, surgery & $6 / 16(37.5)$ \\
\hline Zhao et al., 2012 & 1 & Cutaneous (1) & AmpB, surgery & $0 / 1(0)$ \\
\hline Hatahet et al., 2013 & 1 & Disseminated (1) & AmpB, Mica, surgery & $1 / 1(100)$ \\
\hline Stewart et al., 2014 & 1 & Lungs (1) & Pos, AmpB, Mica, surgery & $0 / 1(0)$ \\
\hline Patel et al., 2014 & 1 & Lungs (1) & Pos, caspofungin & $1 / 1(100)$ \\
\hline Santos et al., 2015 & 2 & Lungs (2) & AmpB, surgery & $2 / 2(100)$ \\
\hline Kursun et al., 2015 & 1 & Rhino (1) & AmpB, surgery & - \\
\hline Palejwala et al., 2016 & 2 & Rhino (2) & AmpB, Pos, mica, Isa, surgery & $2 / 2100$ \\
\hline Vaezi et al., 2016 & 20 & $\begin{array}{l}\text { Rhino (9); lungs (5); } \\
\text { cutaneous (3); disseminated } \\
\text { (2) pseudoaneurysm (1) }\end{array}$ & AmpB, Pos & $10 / 20(50)$ \\
\hline
\end{tabular}

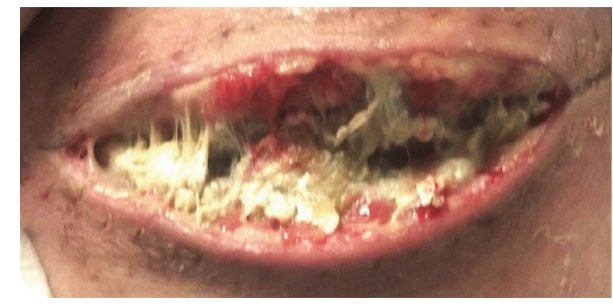

(a)

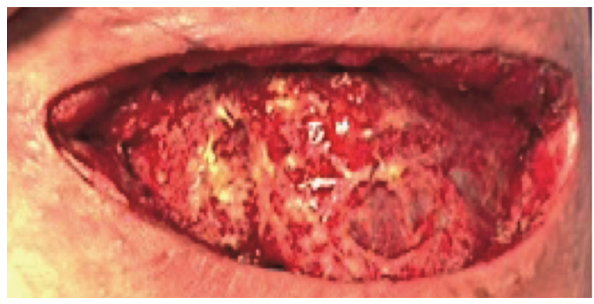

(b)

FIGURE 5: 18-year-old male renal transplant recipient with pulmonary mucormycosis who developed disseminated skin infection with mucormycosis at thoracotomy site (a) and following debridement (b).

with isavuconazonium. An initial strategy of IV liposomal amphotericin B combined with a newer medication, isavuconazonium, was employed along with aggressive surgical debridement. Despite initial efforts, the patient had evidence of recurrent pulmonary mucormycosis with dissemination of the infection to his surgical incision.

The current gold standard for mucormycosis is IV liposomal amphotericin B, surgical debridement, and, if possible, modification of risk factors $[8,10,11]$. Previous case reports have demonstrated the use of posaconazole for salvage therapy. In our study, we used isavuconazonium. Isavuconazonium is a recently developed extended spectrum azole. Studies have demonstrated clinical response to isavuconazonium with a better side effect profile than amphotericin $B$ and posaconazole $[12,13]$. In particular, the incidence of renal injury is significantly lower with isavuconazonium. Aggressive surgical debridement in combination with antifungals has shown to be a mainstay of treatment for mucormycosis. However, despite this knowledge, some case studies reviewed had high mortality rate, approaching $100 \%$ in some studies [12-17]. A summary of published reports from previous studies is presented in Table 1.
There are several important limitations to our study. First, we presented evidence from a single patient. High-quality randomized control trials are needed to further elucidate the use of isavuconazonium. Additionally, it is possible to consider that the first pneumonectomy was inadequate and contributed to the spread of the initial disease to the patient's skin.

In conclusion, we present a rare case of recurrent pulmonary mucormycosis with skin dissemination in an immunocompromised patient that was successfully managed with a combination of surgical treatment and aggressive pharmacologic therapy, including amphotericin $\mathrm{B}$ and isavuconazonium. It is also important to be aware of this possible diagnosis in all immunosuppressed patients and to be well-versed on potential treatment options for recurrent or refractory disease.

\section{Conflicts of Interest}

The authors declare that they have no conflicts of interest regarding the publication of this paper. 


\section{References}

[1] A. Chkhotua, A. Yussim, A. Tovar et al., "Mucormycosis of the renal allograft: case report and review of the literature," Transplant International, vol. 14, no. 6, pp. 438-441, 2001.

[2] M. R. N. Nampoory, Z. U. Khan, K. V. Johny et al., "Invasive fungal infections in renal transplant recipients," Journal of Infection, vol. 33, no. 2, pp. 95-101, 1996.

[3] S. M. Godara, V. B. Kute, K. R. Goplani et al., "Mucormycosis in renal transplant recipients: predictors and outcome," Saudi Journal of Kidney Diseases and Transplantation, vol. 22, no. 4, pp. 751-756, 2011.

[4] M. H. Hatahet, M. Narayanan, C. Cleaves, and R. Zreik, "Disseminated mucormycosis in a patient with recent kidney transplantation: a case report and review of the literature," Case Reports in Nephrology and Urology, vol. 3, no. 1, pp. 58-63, 2013.

[5] B. J. Park, P. G. Pappas, K. A. Wannemuehler et al., "Invasive non-Aspergillusmold infections in transplant recipients, United States, 2001-2006," Emerging Infectious Diseases, vol. 17, no. 10, pp. 1855-1864, 2011.

[6] D. F. Florescu and U. Sandkovsky, "Fungal infections in intestinal and multivisceral transplant recipients," Current Opinion in Organ Transplantation, vol. 20, no. 3, pp. 295-302, 2015.

[7] G. Hamilos, G. Samonis, and D. P. Kontoyiannis, "Pulmonary mucormycosis," Seminars in Respiratory and Critical Care Medicine, vol. 32, no. 6, pp. 693-702, 2011.

[8] N. G. Almyroudis, D. A. Sutton, P. Linden, M. G. Rinaldi, J. Fung, and S. Kusne, "Zygomycosis in solid organ transplant recipients in a tertiary transplant center and review of the literature," American Journal of Transplantation, vol. 6, no. 10, pp. 2365-2374, 2006.

[9] J. A. Vazquez, M. H. Miceli, and G. Alangaden, "Invasive fungal infections in transplant recipients," Therapeutic Advances in Infectious Disease, vol. 1, no. 3, pp. 85-105, 2013.

[10] N. Singh, J. M. Aguado, H. Bonatti et al., "Zygomycosis in solid organ transplant recipients: a prospective, matched case-control study to assess risks for disease and outcome," Journal of Infectious Diseases, vol. 200, no. 6, pp. 1002-1011, 2009.

[11] M. A. Donnelley, E. S. Zhu, and G. R. Thompson, "Isavuconazole in the treatment of invasive aspergillosis and mucormycosis infections," Infection and Drug Resistance, vol. 9, pp. 79-86, 2016.

[12] B. Graves, C. O. Morrissey, A. Wei et al., "Isavuconazole as salvage therapy for mucormycosis," Medical Mycology Case Reports, vol. 11, pp. 36-39, 2016.

[13] S. E. Nagy-Agren, P. Chu, G. J. W. Smith, H. A. Waskin, and F. L. Altice, "Zygomycosis (mucormycosis) and HIV infection: report of three cases and review," Journal of Acquired Immune Deficiency Syndromes and Human Retrovirology, vol. 10, no. 4, pp. 441-449, 1995.

[14] S. Palejwala, T. Zangeneh, S. Goldstein, and G. Lemole, "An aggressive multidisciplinary approach reduces mortality in rhinocerebral mucormycosis," Surgical Neurology International, vol. 7, no. 1, pp. 61-64, 2016.

[15] J. Fisher, C. U. Tuazon, and G. W. Geelhoed, "Mucormycosis in transplant patients," American Surgeon, vol. 46, no. 5, pp. 315322, 1980.

[16] T. Santos, B. Aguiar, L. Santos et al., "Invasive fungal infections after kidney transplantation: a single-center experience," Transplantation Proceedings, vol. 47, no. 4, pp. 971-975, 2015.

[17] A. Patel, E. Bishburg, and S. Nagarakanti, "Mucormycosis in an HIV-infected renal transplant patient: a case report and review of the literature," American Journal of Case Reports, vol. 15, pp. 74-78, 2014. 


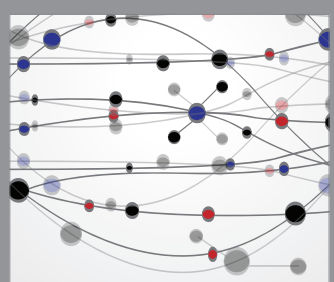

The Scientific World Journal
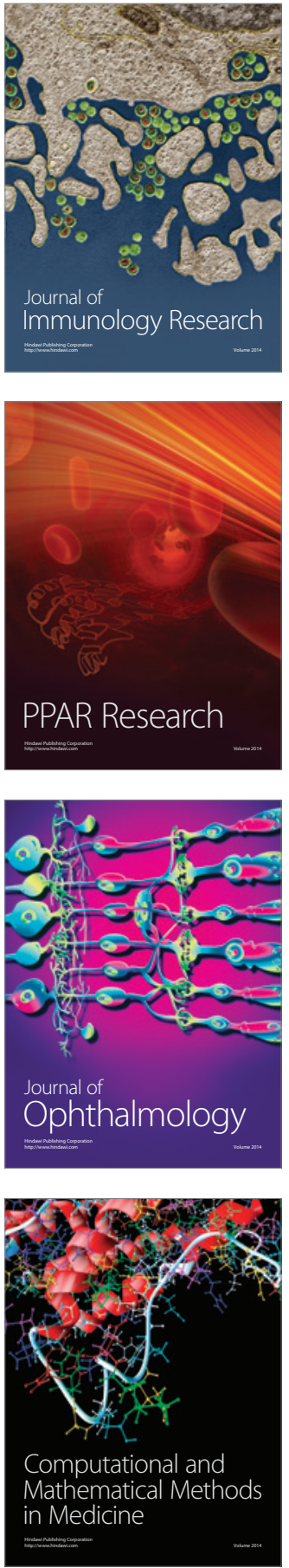

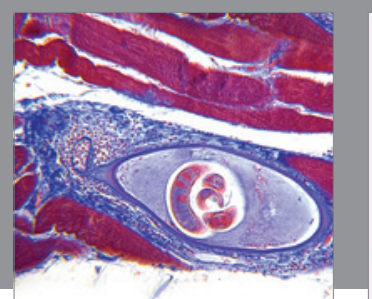

Gastroenterology Research and Practice
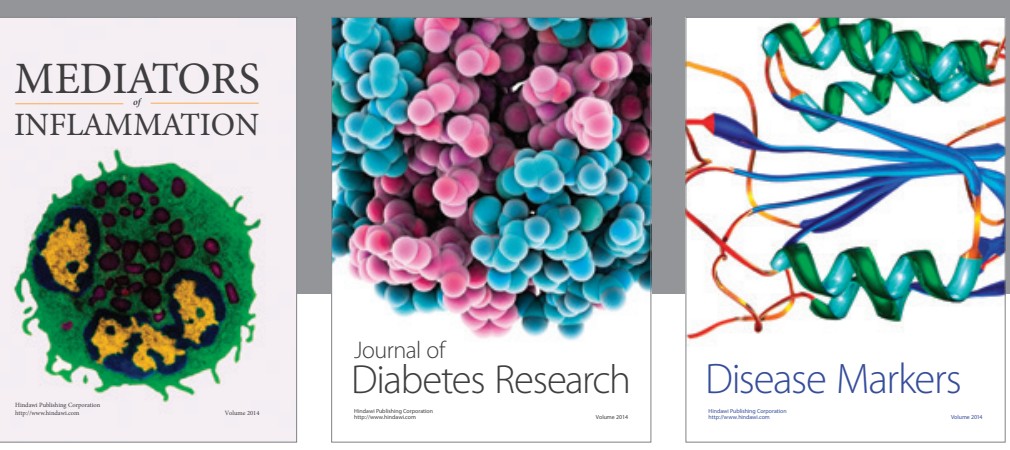

Disease Markers

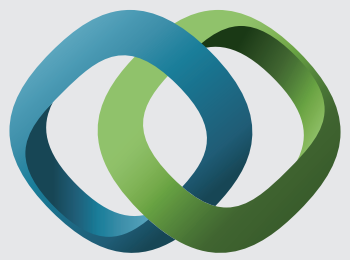

\section{Hindawi}

Submit your manuscripts at

https://www.hindawi.com
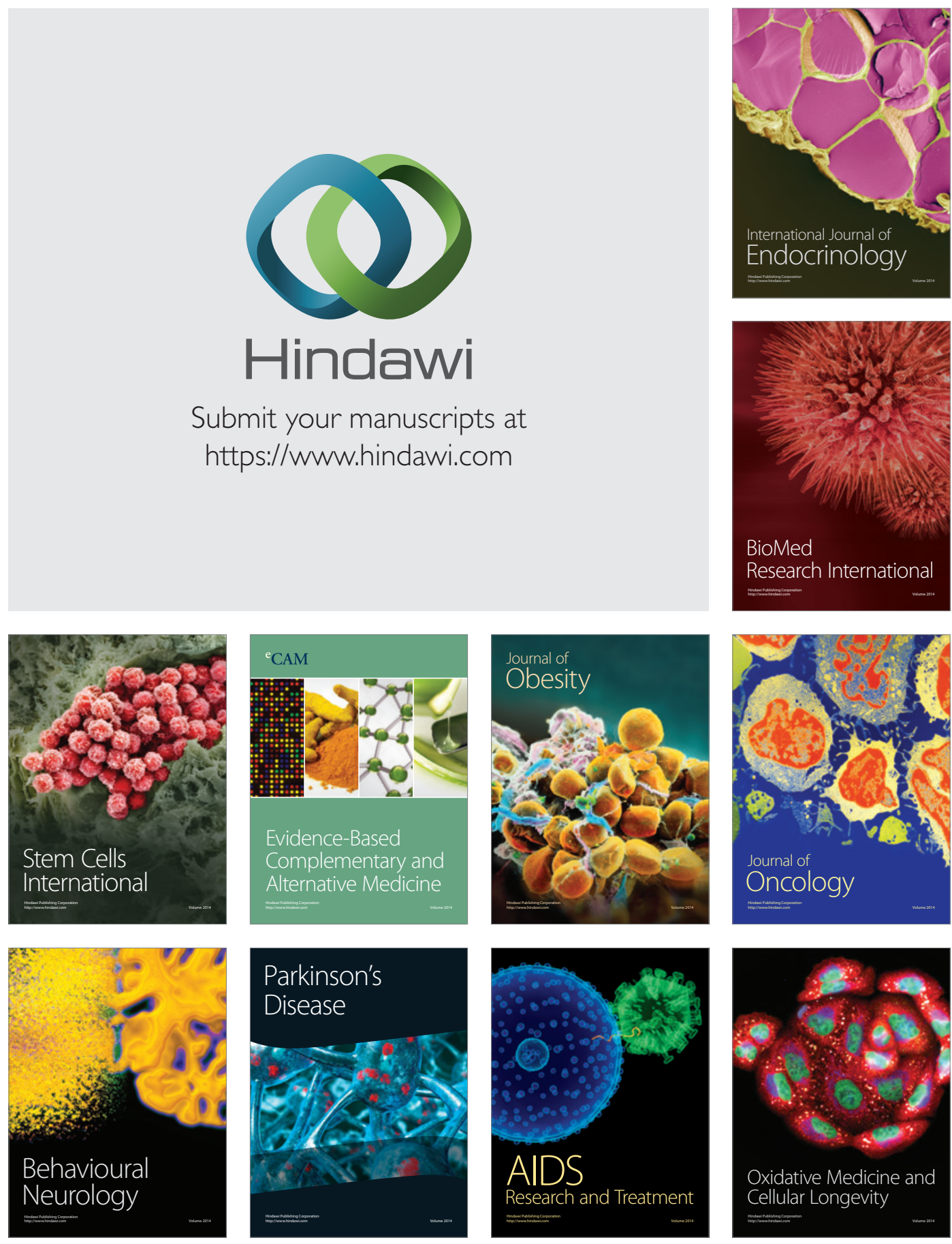